\title{
Domain formation and self-sustained oscillations in quantum cascade lasers ${ }^{\star}$
}

\author{
Tim Almqvist ${ }^{1}$, David O. Winge ${ }^{2}$, Emmanuel Dupont ${ }^{3}$, and Andreas Wacker ${ }^{1, a}$ \\ ${ }^{1}$ Mathematical Physics and NanoLund, Lund University, 22100 Lund, Sweden \\ 2 Synchrotron Radiation Physics and NanoLund, Lund University, 22100 Lund, Sweden \\ ${ }^{3}$ Advanced Electronics and Photonics Research Centre, National Research Council, Ottawa, ON K1A 0R6, Canada
}

Received 31 October 2018 / Received in final form 5 February 2019

Published online 3 April 2019

(C) The Author(s) 2019. This article is published with open access at Springerlink.com

\begin{abstract}
We study oscillations in quantum cascade lasers due to traveling electric field domains, which are observed both in simulations and experiments. These oscillations occur in a range of negative differential resistance and we clarify the condition determining whether the boundary between domains of different electric field can become stationary.
\end{abstract}

\section{Introduction}

Quantum cascade lasers (QCLs) [1,2] have become the most important devices for radiation in the infrared region of the optical spectrum [3] and are also promising for $\mathrm{THz}$ applications [4]. They are based on electronic transitions between quantized states in the conduction band of semiconductor heterostructures, which enables a large flexibility to define the transition energy. QCLs are pumped electrically, where a sequence of scattering and tunneling transition fills the upper laser state and empties the lower one. While such an active module has a typical size of $50 \mathrm{~nm}$, the repetition of a large number of modules (the name-giving cascade) allows to fill the optical waveguide with active material. Here the design is based on the assumption, that the applied bias drops homogeneously over all modules. However, tunneling between quantized states is prone to specific resonances [5] and the appearance of negative differential conductivity (NDC) for fields above the resonance point. In contrast to the resonant-tunneling diode [6], where this property is directly reproduced in the currentbias relation, the cascaded structure of QCLs provides an instability in the homogeneous voltage drop. This situation is analogous to the well-studied domain formation in Gunn diodes [7-9] and superlattices [10-14].

The formation of stationary electrical field domains in QCLs has been observed experimentally by the characteristic saw-tooth structure of the current-bias characteristics $[15,16]$ and a spatially resolved measurement of the bias drop over the sample [17]. Oscillatory behavior has also been reported in the NDC region [18] which was, however,

\footnotetext{
* Contribution to the Topical Issue "Non-Linear and Complex Dynamics in Semiconductors and Related Materials", edited by Kathy Lüdge.

${ }^{a}$ e-mail: Andreas. Wacker@fysik.lu.se
}

interpreted to be determined by the circuit similar to resonant tunneling diodes. Specifically, we consider the THz-QCL with scattering injection discussed in [19]. This structure exhibits characteristic regions of NDC in the simulated current density $J_{\text {hom }}(F d)$ for identical bias drop $F d$ over all modules (so that periodic boundary conditions apply), as shown in Figure 1. Experimentally, current plateaus are observed for biases and currents below threshold. Here we show, both by experiments and simulations, that oscillating electric field domains occur in our device, similar to the case of superlattices [20], and discuss the conditions for their occurrence.

\section{Simulating domain formation}

In order to simulate domain formation, we assume that the sheet electron density $n_{i}$ of each module is essentially confined in a narrow region before the thickest barrier. This is confirmed by our non-equilibrium Green's function (NEGF) simulations as shown in Figure 2. (For details, see [21], where all parameters are givens. As the only difference we use a slightly increased $\mathrm{rms}$ height $\eta=3 \AA$ for the interface roughness here, which appears to match better with the experimental results. We have no direct experimental information regarding the quality of interfaces.) The same location of charges was actually deduced experimentally [17]. The current to the next module is then essentially determined by the bias drop $F_{i} d$, where $F_{i}$ is the average electric field between the electron densities located in module $i$ and $i+1$ and $d=36.12 \mathrm{~nm}$ is the thickness of a single module. Following a common approach in superlattices [13], this current density is approximated by

$$
J_{i \rightarrow i+1}=J_{\mathrm{hom}}\left(F_{i} d\right) \frac{n_{i}-n_{i+1} \mathrm{e}^{-e F_{i} d / k_{\mathrm{B}} T}}{N_{d}-N_{d} \mathrm{e}^{-e F_{i} d / k_{\mathrm{B}} T}},
$$




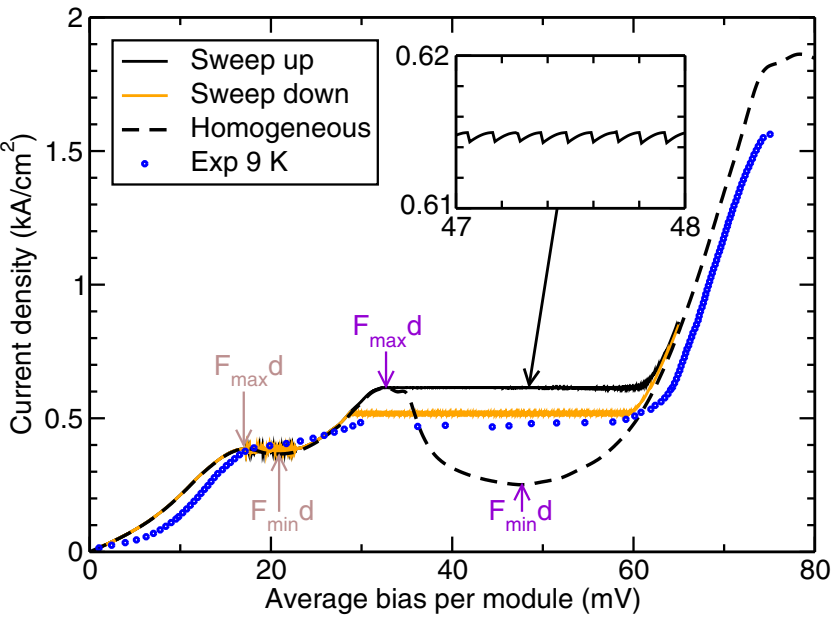

Fig. 1. Simulated current-bias relation with domain formation for a phonon temperature of $77 \mathrm{~K} . J_{\text {hom }}(F d)$ (dashed line) is calculated by our NEGF simulation and shows two NDC regions between fields $F_{\max }$ and $F_{\min }$, which denote maxima and minima of current. The full black/orange line shows the result with domain formation upon sweep-up/down of the bias, respectively. An enlarged section for sweep up is shown in the inset. The blue dots show corresponding experimental data.

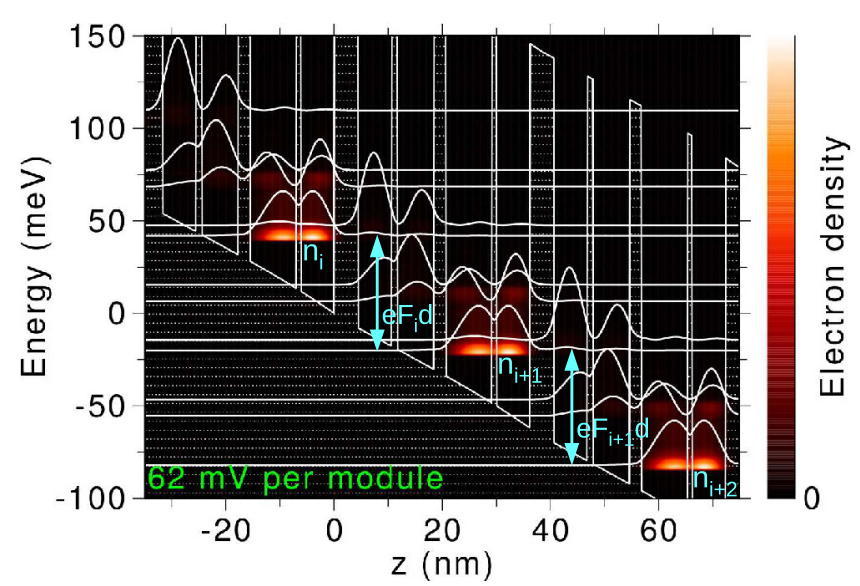

Fig. 2. Simulated electron density based on a homogeneous bias drop and description of the notation for electron sheet densities $n_{i}$ and potential drop $F_{i} d$.

which assumes that the current density is essentially proportional to the electron density $n_{i}$ in the injecting module and is reduced by a backward current from thermal excitations in the receiving module $i+1$. This expression is normalized by the sheet doping density $N_{d}=3.25 \times$ $10^{10} \mathrm{~cm}^{-2}$ per module, so that the homogeneous current is recovered for $n_{i}=n_{i+1}=N_{d}$. Then the continuity equation provides

$$
e \frac{\mathrm{d} n_{i}}{\mathrm{~d} t}=J_{i-1 \rightarrow i}-J_{i \rightarrow i+1} \quad \text { for } i=1, \ldots N
$$

where $N=276$ is the number of modules. $e$ is the positive elementary charge and we redefine the sign of electric field and current density, so that their signs match the forces and velocity of carriers, respectively. In equation (2) we need the boundary currents, which we approximate as

$$
J_{0 \rightarrow 1}=\sigma F_{0} \quad J_{N \rightarrow N+1}=\sigma F_{N} \frac{n_{N}}{N_{d}}
$$

with a phenomenological boundary conductivity $\sigma$, as justified for the Gunn diode in reference [22]. These Ohmic boundary conditions are common for superlattices, see $[13,23]$ for a more detailed discussion. Unless mentioned otherwise, we use $\sigma / d=50 \mathrm{~A} /\left(\mathrm{cm}^{2} \mathrm{mV}\right)$, which is steeper than the slopes in $j_{\text {hom }}(F d)$. This mimics a good Ohmic contact between the QCL heterostructure and the substrate with an adjacent highly doped $\left(8 \times 10^{17} / \mathrm{cm}^{3}\right)$ GaAs layer implying a small bias drop $F_{0} d$. For given electron densities $n_{i}$, the electric fields are obtained from the total bias $U$ dropping over the QCL

$$
U=\sum_{i=0}^{N} F_{i} d
$$

and Poisson's equation

$$
F_{i}-F_{i-1}=\frac{e}{\epsilon_{0} \epsilon_{r}}\left(n_{i}-N_{d}\right) \quad \text { for } i=1, \ldots N
$$

where $\epsilon_{r}=13$ is the average relative dielectric constant. For fixed bias $U$, this model has been applied for QCLs in [16]. Here we consider also the operation by a fixed bias source $U_{0}$ via a serial load resistance $R$. Following [24] we find

$$
\begin{aligned}
\frac{\mathrm{d} U}{\mathrm{~d} t} & =\frac{1}{C_{\mathrm{QCL}}+C_{p}}\left(\frac{U_{0}-U}{R}-I\right) \\
\text { with } I & =\frac{A}{N+1} \sum_{i=0}^{N} J_{i \rightarrow i+1} .
\end{aligned}
$$

Here $C_{\mathrm{QCL}}=\epsilon_{r} \epsilon_{0} A /(N+1) d$ is the geometrical capacitance of the QCL structure with $A=0.144 \mathrm{~mm}^{2}$ and $C_{p}$ is an additional parallel capacitance, which we actually set to zero here.

The set of equations (1), (2), (5) can be viewed as the discretization of a drift-diffusion model combined with Poisson's equation (as appropriate for the Gunn effect [8]). Here, the drift velocity $v(F)$ and the diffusion coefficient $D(F)$ are given by

$$
v(F)=\frac{d J_{\mathrm{hom}}(F d)}{e N_{d}} \quad \text { and } D(F)=\frac{d^{2} J_{\mathrm{hom}}(F d)}{e N_{d}\left(\mathrm{e}^{e F d / k_{\mathrm{B}} T}-1\right)},
$$

respectively, which satisfy the Einstein relation in the linear regime for low fields. This allows for analytic insight into the dynamics of wave-fronts $[23,25]$. However, for many features, such as the existence of stable field domains in a finite range of currents, the discreteness due to the semiconductor heterostructure is essential. 


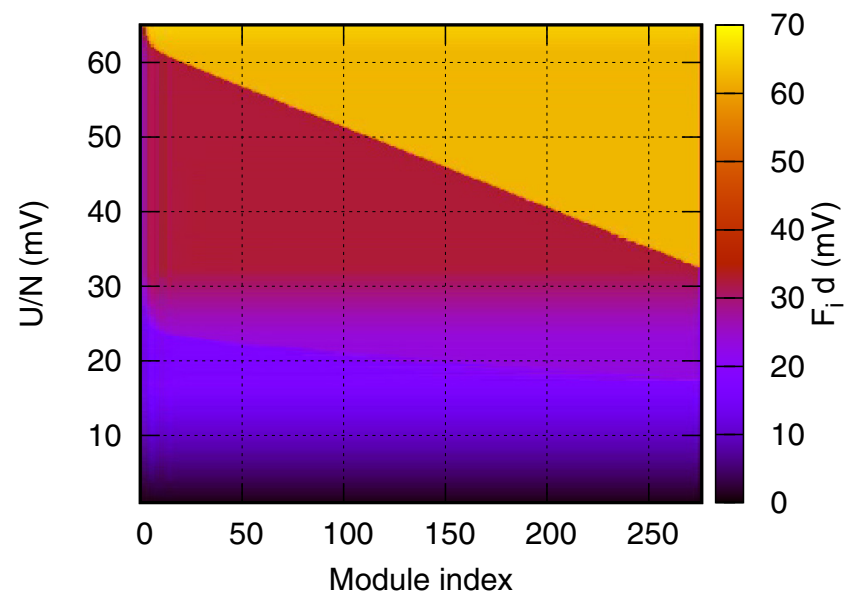

Fig. 3. Simulated field distribution (in color scale) as a function of position and total bias, obtained by sweeping up the bias. (For $U / N \sim 20 \mathrm{mV}$ the domain boundary oscillates in time and typical snapshots are displayed.)

\section{Simulation results}

Figure 1 shows the simulated current bias relations using a fixed bias $U$. Upon sweeping up the bias, we find two current plateaus; after the first current peak around an average bias of $17 \mathrm{mV}$ per module and also after the second current peak around an average bias of $33 \mathrm{mV}$ per module. The current depends on the sweep direction, a common feature, which has been discussed for superlattices in detail, where multi-stability was observed [12]. The corresponding distribution of the electric field is shown in Figure 3. Within the plateau regions, there are two domains with specific electric fields. An increase of bias is reflected in a shift of the domain boundary, where the high-field domain expands.

For the first plateau, the simulations provide actually oscillatory behavior as shown in Figure 4. These are due to moving domain boundaries (also referred to as fronts), where the excess charge travels along the direction of electron motion. For $J=300 \mathrm{~A} / \mathrm{cm}^{2}$ we obtain an average drift velocity of $J /\left(N_{d} e\right)=60$ modules/ns, which is comparable to the average front velocity of 50 modules/ns observed in Figure 4a. During the motion of the domain boundary, the electric fields in both domains need to increase in order to maintain the fixed bias. Eventually, the electric field in the low-field domain reaches the NDC region (e.g. at $t \approx 3 \mathrm{~ns}$ ) and becomes unstable. Thus, a new accumulation layer is formed within a fraction of a nanosecond, which then starts its motion like the one before. If the sample is operated via a load resistor, the bias $U$ is actually oscillating as shown in Figure 5. In this case the oscillation frequency is reduced.

Such oscillations are generic for extended systems with NDC and have been well-studied for the Gunn Diode and superlattices $[9,23]$. In continuous systems, such as the Gunn diode, any charge accumulation typically travels due to the drift velocity (albeit the actual speed may differ [8]) and thus stationary fronts are only possible in the vicinity of the contacts. However, for discrete systems,
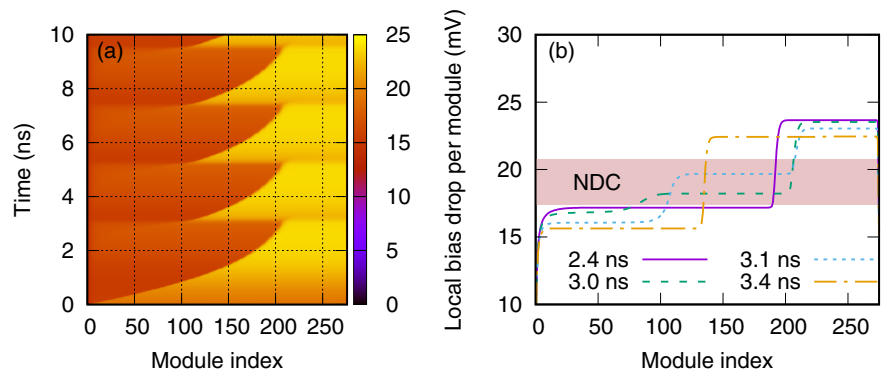

Fig. 4. (a) Simulated field distribution (in color scale) as a function of position and time for a fixed average bias $U / N=19 \mathrm{mV}$. Panel (b) shows the field distribution at particular times.

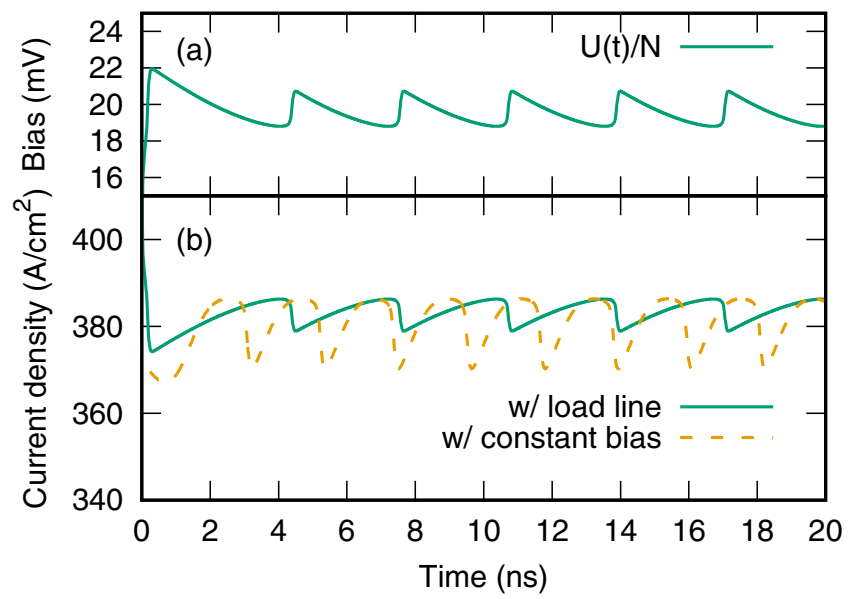

Fig. 5. (a) Bias and (b) current density for simulations with $R=50 \Omega$ and $U_{0}=33 \mathrm{~V}$ in comparison with the bias controlled case (dashed) with $U / N=19 \mathrm{mV}$ as considered in Figure 4.

such as superlattices and QCLs, these fronts can become stationary for a finite range of currents $[11,12,15,26]$, so that the oscillations disappear. Figure 6 shows the electron density distribution close to the domain boundary in both plateaus. For the first plateau, see Figure 6b, the excess electron density $n_{\text {excess }}=\epsilon_{0} \epsilon_{r}\left(F_{\text {high }}-F_{\text {low }}\right) / e$, required to change the field between both domains, is spread out over several modules, which resembles the continuous case of the Gunn diode. In contrast, for the second plateau, see Figure 6a, a large part of the excess density is essentially located in one module and is trapped by the heterostructure, so that a stationary front appears [27]. This situation should be stable, if the NDC region is crossed within one module. Then at least the part $n_{\mathrm{NDC}}=\epsilon_{0} \epsilon_{r}\left(F_{\min }-F_{\max }\right) / e$ of $n_{\text {excess }}$ is located within one module, where $F_{\max } /$ min denote the position of the current maximum/minimum at the borders of the NDC region, respectively, as indicated in Figure 1. In order for this to happen, the current in a module with the excess density must match the current $j$ of the other modules. Using the approximation for the current (1) under neglect of the backward currents [i.e. the terms with $\mathrm{e}^{-e F_{i} d / k_{\mathrm{B}} T}$ in Eq. (1)], this implies the condition for 

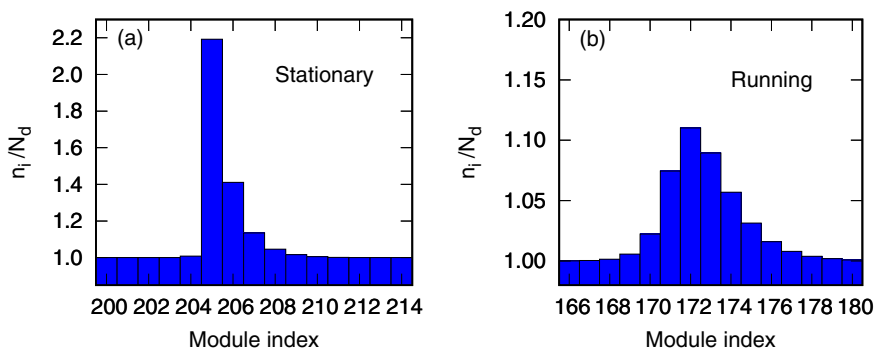

Fig. 6. Charge distribution of accumulation layer for domains at an average bias $U / N=40 \mathrm{mV}$ (left with stationary domain) and $19 \mathrm{mV}$ (right with running domain).

stationary accumulation fronts

$$
\left(\frac{n_{\mathrm{NDC}}}{N_{d}}+1\right) j_{\min } \lesssim j
$$

where $j_{\min }$ is the minimum current in the NDC region. A slightly stronger bound has been proven in equation (18) of [26] (also including the backward currents), which justifies the heuristic argument given above. As the low-field domain is only possible for $j<j_{\max }$, where $j_{\max }$ is the peak current before the NDC region we find the criterion for stationary domains with accumulation regions

$$
\left(\frac{n_{\mathrm{NDC}}}{N_{d}}+1\right) \lesssim \frac{j_{\max }}{j_{\min }}
$$

A thorough proof for the stability of stationary domains under this criterion had been provided in [27] without backward current, and follows more generally from [26]. Based on the homogeneous current-field relations in Figure 1 , we find $n_{\mathrm{NDC}} / N_{d}+1=1.25$ for the first NDC region, which surpasses the ratio between peak and valley current $j_{\max } / j_{\min } \approx 1.05$. This agrees with finding only traveling fronts and oscillatory behavior in this plateau region. For the second plateau $n_{\mathrm{NDC}} / N_{d}+1=1.92$ is lower than $j_{\max } / j_{\min } \approx 2.44$ and stationary fronts are possible for currents above $j=1.92 j_{\min } \approx 490 \mathrm{~A} / \mathrm{cm}^{2}$ according to equation (7). This value agrees well with the minimal current density $j_{\min }^{\text {stat }}=515 \mathrm{~A} / \mathrm{cm}^{2}$ for the domain states upon sweeping down the bias in Figure 1. In order to compare with experiment, it is important to realize that equation (1) is an approximation and that the minimum current density $j_{\min }$ is difficult to access. Furthermore, growth imperfections can shift the range of doping where oscillating field domains are observed in simulations for superlattices [28]. However, the condition (8) should reflect the most important trend, that stationary field domains require large doping densities and a pronounced peak to valley ratio.

\section{Experimental results}

The experimental data displayed in Figure 1 show a clear current plateau in the second simulated NDC region (between 34 and $60 \mathrm{mV}$ ), while the current is

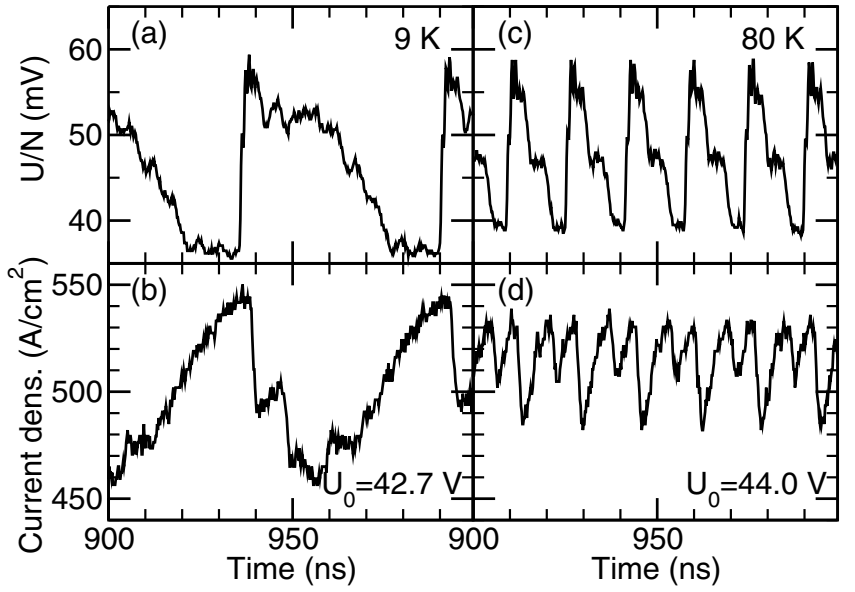

Fig. 7. Experimentally detected oscillations at a heat sink temperature of $9 \mathrm{~K}$ for a bias $U_{0}=42.7 \mathrm{~V}(\mathrm{a}, \mathrm{b})$ and at $80 \mathrm{~K}$ for $U_{0}=44 \mathrm{~V}(\mathrm{c}, \mathrm{d})$. The device is operated via a load resistance with $R=40.7 \Omega$. Note that current and bias have been recorded with different cables connecting the cryostat, and that the frequency response of the current and voltage probes are different too, which all together can result to time delays of a few ns between the two signals.

monotonously increasing in the first simulated NDC region around $20 \mathrm{mV}$. Using a significantly different setup, reference [17] reported the occurrence of stationary electric field domains in the second region but a homogeneous bias drop in the first region. This indicates, that the first (weak) NDC region seen in our simulations is not visible in the device. (It also vanishes in the simulations for an increased roughness $\eta=4 \AA$ or an elevated temperature of $150 \mathrm{~K}$.) On the other hand, NDC and the formation of electric field domains is manifest in the pronounced second simulated NDC region.

Our measurements are based on $2 \mu$ s long pulses and the device is operated via a load resistance of $40.7 \Omega$ with a similar setup as described in [29]. In contrast to [29], the laser bar studied here is indium soldered to a $\mathrm{Si}$ carrier with electrodes of negligible resistivity, which simplifies the data analysis. Using a $1 \mathrm{GHz}$ bandwidth oscilloscope, we observe oscillatory behavior in the bias as shown in Figures $7 \mathrm{a}$ and $7 \mathrm{c}$. For a heatsink temperature of $80 \mathrm{~K}$, regular oscillations are observed for external bias $41.4 \mathrm{~V}<$ $U_{0}<48.5 \mathrm{~V}$. From $48.5 \mathrm{~V}$ to $49.5 \mathrm{~V}$ irregular oscillations are observed, in the sense that the FFT intensity spectrum collapses. While this might indicate some chaotic scenario, we cannot exclude, that these features are triggered by the noise of the input pulses. Above $49.5 \mathrm{~V}$, few sporadic short surges of voltage are still observed indicating some voltage instabilities at the exit of the plateau. At the very beginning of the plateau, from 41.4 to $42.1 \mathrm{~V}$, high amplitude oscillations at fundamental frequency $\sim 27.6 \mathrm{MHz}$ are observed. At $42.1 \mathrm{~V}$, as intense but faster oscillations appear at $\sim 59 \mathrm{MHz}$. As the external bias further increased, the frequency of oscillations increases slightly and its amplitude decreases monotonously. When the regular oscillations vanishes at $48.5 \mathrm{~V}$, the frequency has shifted to $\sim 65.1 \mathrm{MHz}$. The range of external voltage 
where the voltage oscillations or instabilities occur is very consistent with the observation of the current plateau. For a heatsink temperature of $9 \mathrm{~K}$, the observed frequencies are lower and the oscillations are not found at all operation points within the plateau.

Concomitant oscillations of the total current (QCL plus voltage probe) measured at the load resistor were also observed and are displayed in Figures $7 \mathrm{~b}$ and $7 \mathrm{~d}$. The current transformer used has a bandwidth of $200 \mathrm{MHz}$, which limits the resolution here.

\section{Simulations with reduced contact conductivity}

The experimental observation of oscillations within the second NDC region of Figure 1 does not agree with the simulated stationary domains discussed in Section 3, which are expected due to the stability criterion (8). These stationary field domain distributions show an electron accumulation layer separating the low-field domain close to the injecting contact and the high-field domain at the receiving contact. ${ }^{1}$ Such a field distribution is only possible if the contact field $F_{0}$ is small at the relevant currents, as otherwise a high-field domain is present at the injecting contact. As discussed in Section 3, the stationary domain states require current-densities above $j_{\min }^{\text {stat }}=515 \mathrm{~A} / \mathrm{cm}^{2}$ as a consequence of the criterion (7). In the following we use a low contact conductivity $\sigma / d=$ $12 \mathrm{~A} /\left(\mathrm{cm}^{2} \mathrm{mV}\right)$, which would reflect a significant offset between the elctrochemical potential in the contacting layer and the levels in QCL structures. The chosen value of $\sigma$ implies $F_{0} d \geq 43 \mathrm{mV}$ for $j_{0 \rightarrow 1} \geq j_{\min }^{\text {stat }}$, which is close to $F_{\min } d$ at the end of the NDC region. Thus the low-field domain cannot exist at the injecting contact for $j \gtrsim j_{\min }^{\text {stat }}$ and the stable domain configurations obtained for the second NDC region in Section 3 cannot exist. As a consequence we find oscillatory behavior similar to the experimental observation.

Figure 8 shows oscillatory behavior for a fixed average bias $U / N=40 \mathrm{mV}$, i.e. in the region of the second plateau. Figure 8b shows a conventional electric field domain distribution at 11 ns. Figure 8a shows that the current density is below $j_{\mathrm{min}}^{\text {stat }}$ and thus the distribution is not stationary but travels to the receiving contact. The constant bias provides a raise in the fields and an increase in current. At $12 \mathrm{~ns}$, the field $F_{0}$ becomes so large that a high-field domain forms at the injecting contact and starts traveling towards the receiving contact. Simultaneously, the fields drop in all domains and due to the drop of current, the injecting contact can sustain a low-field domain again after $12.5 \mathrm{~ns}$. Afterwards a characteristic period with constant current arises, where the two accumulation fronts travel with half the velocity of the depletion front in between. This scenario is explained in detail for superlattice in reference [13]. Around 15 ns one accumulation

\footnotetext{
${ }^{1}$ Domain distributions with a depletion layer, which have the high-field domain at the injecting contact, are also possible. In order to be stationary, a condition similar to equation (8) exists [13], which however requires a substantially higher doping and is not satisfied for our device.
}

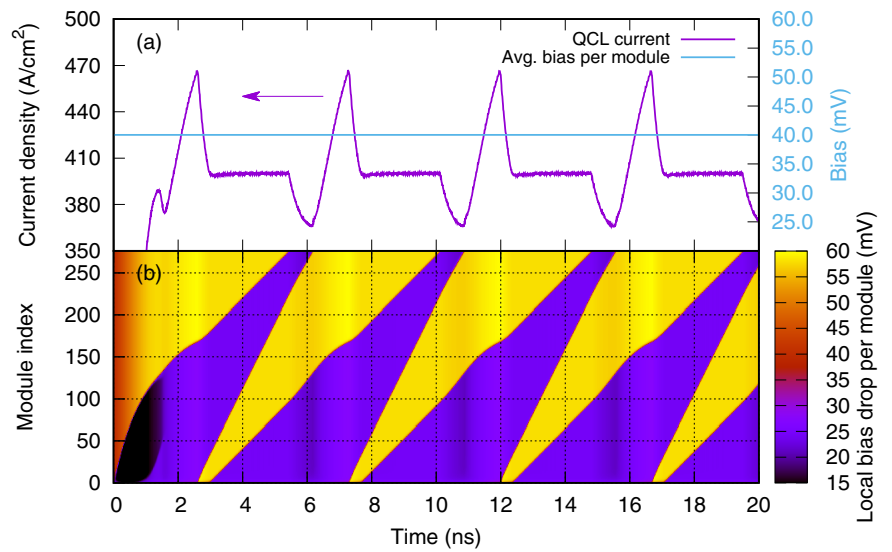

Fig. 8. Simulated oscillations in the second plateau for a fixed bias per module of $40 \mathrm{mV}$ applying a reduced contact conductivity $\sigma / d=12 \mathrm{~A} /\left(\mathrm{cm}^{2} \mathrm{mV}\right)$.

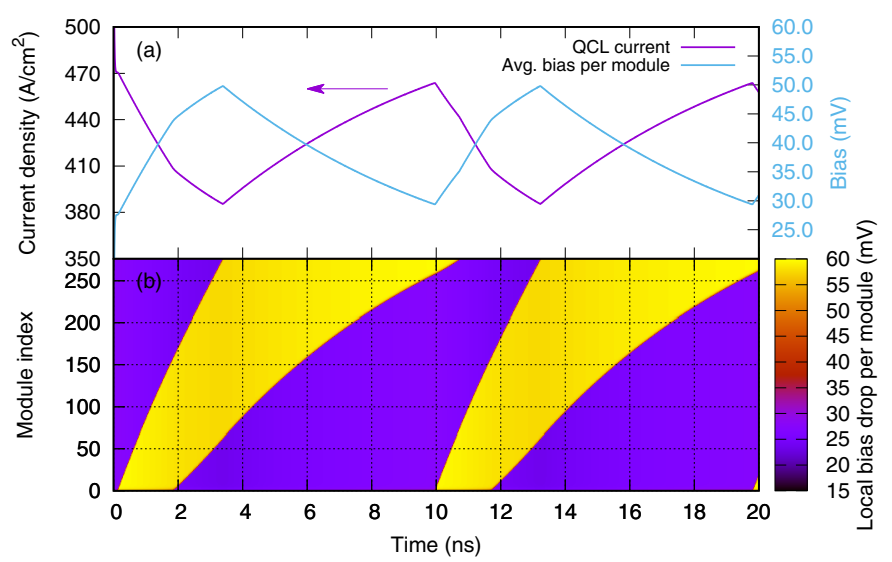

Fig. 9. Simulated oscillations in the second plateau for operating via a load resistance of $50 \Omega$ and $U_{0}=41.5 \mathrm{~V}$ applying a reduced contact conductivity $\sigma / d=12 \mathrm{~A} /\left(\mathrm{cm}^{2} \mathrm{mV}\right)$.

front and subsequently the depletion front vanish at the receiving contact and the cycle is repeated.

Figure 9 shows similar domain oscillations under circuit conditions with a load resistor. The frequency is reduced and the signals are altered. Comparing with the electric field distribution in Figure 9b, we find that the maxima and minima in current from Figure 9a are related to the creation of a depletion front at the injection contact and its vanishing at receiving contact, respectively. The corresponding creation and vanishing of the accumulation front is associated with a smaller increase and decrease of slope in the current signal, respectively. The bias behaves precisely the opposite way. Upon varying $U_{0}$ we find similar results over the entire second NDC region with average current densities around $430 \mathrm{~A} / \mathrm{cm}^{2}$. This current plateau (not shown) agrees excellently with the experimental data in Figure 1 and the simulated oscillation frequencies are comparable (about a factor two larger) to the experiment (at $80 \mathrm{~K}$ ). However, the particular shapes of the current and bias signal differ, which might be related to more intricate boundary currents $j_{0 \rightarrow 1}\left(F_{0}\right)$ or to details in the 
circuit such as a parallel capacitance $C_{p}$ not accounted for in our simulations.

\section{Conclusion and discussion}

We demonstrated both by simulations and experimentally, that oscillating electric field domains are possible in QCLs. Stationary domains are favored by high doping, a large peak to valley ratio $j_{\text {peak }} / j_{\text {min }}$ in the NDC region and a small excess charge between the domains as quantified by equation (8). Furthermore the injecting contact needs to allow for the presence of a low-field domain at its vicinity for current densities above the minimal current $j_{\min }^{\text {stat }}$ for stationary domain states. $j_{\text {min }}^{\text {stat }}$ can be estimated by equation (7). While the simulated current-bias relations agree well with the experimental data, we did not obtain full agreement regarding the details of the oscillations. In particular, the simulations provide domain oscillations in the first NDC region, which appear to be absent in the experiment. This might be related to a higher background current in the experiment, which counteracts the weak NDC feature. Furthermore, the oscillation signals in the second NDC region differ, which we can not explain now. Finally, we want to point out, that very recently some of us observed domain oscillations in a different QCL, which also persisted after the onset of lasing both experimentally and by simulations [29].

Financial support from the Swedish Research Council (grant 2017-04287) and Nanolund is gratefully acknowledged.

\section{Author contribution statement}

Tim Almqvist did the simulations and Emmanuel Dupont the measurements presented here. The numerical codes were developed by Tim Almqvist, David Winge, and Andreas Wacker, who also guided the work. All the authors were involved in the preparation of the manuscript and approved the final version.

Open Access This is an open access article distributed under the terms of the Creative Commons Attribution License (http://creativecommons.org/licenses/by/4.0), which permits unrestricted use, distribution, and reproduction in any medium, provided the original work is properly cited.

\section{References}

1. J. Faist, F. Capasso, D.L. Sivco, C. Sirtori, A.L. Hutchinson, A.Y. Cho, Science 264, 553 (1994)
2. J. Faist, Quantum Cascade Lasers (Oxford University Press, Oxford, 2013)

3. M. Razeghi, W. Zhou, S. Slivken, Q.Y. Lu, D. Wu, R. McClintock, Appl. Opt. 56, H30 (2017)

4. B. Röben, X. Lü, M. Hempel, K. Biermann, L. Schrottke, H.T. Grahn, Opt. Express 25, 16282 (2017)

5. F. Capasso, K. Mohammed, A.Y. Cho, Appl. Phys. Lett. 48, 478 (1986)

6. L.L. Chang, L. Esaki, R. Tsu, Appl. Phys. Lett. 24, 593 (1974)

7. J.B. Gunn, IBM J. Res. Dev. 8, 141 (1964)

8. H. Kroemer, IEEE Trans. Electr. Dev. ED-13, 27 (1966)

9. M.P. Shaw, V.V. Mitin, E. Schöll, H.L. Grubin, The Physics of Instabilities in Solid State Electron Devices (Plenum Press, New York, 1992)

10. L. Esaki, L.L. Chang, Phys. Rev. Lett. 33, 495 (1974)

11. L.L. Bonilla, J. Galán, J.A. Cuesta, F.C. Martínez, J.M. Molera, Phys. Rev. B 50, 8644 (1994)

12. J. Kastrup, H.T. Grahn, K. Ploog, F. Prengel, A. Wacker, E. Schöll, Appl. Phys. Lett. 65, 1808 (1994)

13. A. Wacker, Phys. Rep. 357, 1 (2002)

14. L.L. Bonilla, H.T. Grahn, Rep. Prog. Phys. 68, 577 (2005)

15. S.L. Lu, L. Schrottke, S.W. Teitsworth, R. Hey, H.T. Grahn, Phys. Rev. B 73, 033311 (2006)

16. M. Wienold, L. Schrottke, M. Giehler, R. Hey, H.T. Grahn, J. Appl. Phys. 109, 073112 (2011)

17. R.S. Dhar, S.G. Razavipour, E. Dupont, C. Xu, S. Laframboise, Z. Wasilewski, Q. Hu, D. Ban, Sci. Rep. 4, 7183 (2014)

18. C. Sirtori, H. Page, C. Becker, V. Ortiz, IEEE J. Quantum Elect. 38, 547 (2002)

19. E. Dupont, S. Fathololoumi, Z.R. Wasilewski, G. Aers, S.R. Laframboise, M. Lindskog, S.G. Razavipour, A. Wacker, D. Ban, H.C. Liu, J. Appl. Phys. 111, 073111 (2012)

20. J. Kastrup, R. Klann, H.T. Grahn, K. Ploog, L.L. Bonilla, J. Galán, M. Kindelan, M. Moscoso, R. Merlin, Phys. Rev. B 52, 13761 (1995)

21. D.O. Winge, M. Franckié, A. Wacker, J. Appl. Phys. 120, $114302(2016)$

22. H. Kroemer, IEEE Trans. Electr. Dev. 15, 819 (1968)

23. L.L. Bonilla, S.W. Teitsworth, Nonlinear Wave Methods for Charge Transport (Wiley-VCH, Weinheim, 2010)

24. A. Wacker, E. Schöll, J. Appl. Phys. 78, 7352 (1995)

25. L. Bonilla, SIAM J. Appl. Math. 57, 1588 (1997)

26. A. Carpio, L.L. Bonilla, A. Wacker, E. Schöll, Phys. Rev. E 61, 4866 (2000)

27. A. Wacker, M. Moscoso, M. Kindelan, L.L. Bonilla, Phys. Rev. B 55, 2466 (1997)

28. A. Wacker, G. Schwarz, F. Prengel, E. Schöll, J. Kastrup, H.T. Grahn, Phys. Rev. B 52, 13788 (1995)

29. D.O. Winge, E. Dupont, A. Wacker, Phys. Rev. A 98, 023834 (2018) 